\title{
Ecotalleres para la planificación curricular con enfoque ambiental
}

\author{
Olga Marivi Olivares Huamanchumo \\ marivicita25@gmail.com \\ ORCID: $\underline{0000-0002-9070-2748}$ \\ Dr. Groberti Alfredo Medina Corcuera \\ grome@ucvvirtual.edu.pe \\ ORCID: $\underline{0000-0003-4035-157 \mathrm{X}}$ \\ Universidad Cesar Vallejo. \\ Chimbote - Perú
}

\section{RESUMEN}

El objetivo del estudio fue implementar, aplicar y evaluar los Ecotalleres para mejorar el conocimiento sobre planificación curricular con enfoque ambiental en docentes de inicial de la Provincia del Santa - 2021. La investigación fue de tipo Aplicada, Pre experimental, el instrumento: un cuestionario, la muestra 65 docentes. Los ecotalleres se implementaron con las estrategias activas, participativas y reflexivas. Durante la aplicación, 95\% de maestras asisten continuamente y $62 \%$ alcanzan nivel Logrado en los trabajos colaborativos. Antes de la aplicación de los Ecotalleres $83 \%$ de docentes se hallaban En inicio. Después de la aplicación las participantes alcanzaron niveles superiores de desempeño. Al comparar el conocimiento de las maestras antes y después de los Ecotalleres: el grupo de $83 \%$ disminuyó a 6.15\%; el 15\% que se encontraba En proceso avanzó a 32.31\%; el 2\% de nivel Esperado alcanzó el 33.85\% y, de ninguna docente en el nivel destacado en el pre test, en el post test alcanzaron el 27.69\%. Además, con los resultados de la Prueba de rangos con signo de Wilcoxon, se concluye que: la aplicación de los Ecotalleres influye significativamente en el nivel de conocimiento de la planificación curricular con enfoque ambiental de las educadoras, constituyéndose en propuesta exitosa.

Palabras clave: ecotaller; planificación; enfoque ambiental. 


\title{
Eco-workshops for curricular planning with an environmental focus
}

\begin{abstract}
The objective of the study was to implement, apply and evaluate the Eco-workshops to improve the knowledge about curricular planning with an environmental focus in preschool teachers of the Province of Santa - 2021. The research was Applied, Preexperimental, the instrument: a questionnaire, the sample 65 teachers. The eco-workshops were implemented with active, participatory and reflective strategies. During the application, $95 \%$ of teachers attend continuously and 62\% reach the Achieved level in collaborative works. Before the application of the Ecotalleres $83 \%$ of teachers were in the beginning. After the application, the participants reached higher levels of performance. When comparing the knowledge of the teachers before and after the Eco-workshops: the $83 \%$ group decreased to $6.15 \%$; the $15 \%$ that was in process advanced to $32.31 \%$; The $2 \%$ of the Expected level reached $33.85 \%$ and, of no teacher in the outstanding level in the pre-test, in the post-test they reached $27.69 \%$. In addition, with the results of the Wilcoxon signed rank test, it is concluded that: the application of the Eco-workshops significantly influences the educators' level of knowledge of curricular planning with an environmental focus, becoming a successful proposal.
\end{abstract}

Keywords: ecotaller; planning; environmental approach.

Artículo recibido: 30 noviembre. 2021 Aceptado para publicación: 29 diciembre 2021 Correspondencia: marivicita25@gmail.com Conflictos de Interés: Ninguna que declarar 


\section{INTRODUCCIÓN}

En el mes de marzo del 2019, el Programa de Naciones Unidas por el Medio Ambiente, cuyas siglas son: PNUMA presentó un extenso informe alertando a la comunidad Mundial sobre el incumplimiento del Acuerdo de Paris, el que es: reducir las emanaciones de los gases que condicionan la consecuencia de invernadero de $40 \%$ a $70 \%$ en los periodos de 2010 a 2050. Esperando que para el 2070 este efecto deberá haberse reducido a cero. Destacando que, si bien alcanzar esta meta tiene un costo de 19,5 billones de euros, el ahorro en salud mundial debido a muertes y enfermedades que se evitarían serían 47,8 billones de euros (Programa Mundial de Educación por el Ambiente UNESCO-PNUMA, 2019). Este problema Ambiental que aqueja a nuestro planeta es un tema que se observa con muy poca conciencia, las personas están ocupadas disfrutando de los beneficios del progreso, del avance de la tecnología, sin percatarse de los residuos que ésta genera en su producción o al desecharlos, entre otras consecuencias contaminantes (Sarria y Gallo, 2016). En esa misma línea están las diversas actividades económicas extractivas (Arteaga-Cruz, Mukhopadhyay, Shannon, Nidhi y Jailer, 2021)

Son situaciones que no se van a solucionar con acuerdos de alto nivel o normas (Huynh, 2020). Se necesita que el proceso de concientización reflexiva de lo que está ocurriendo y el consecuente cambio, se dé dentro de cada persona, ahí está el gran desafío (Silva y Passos, 2018). Desafío que apunta a la educación por cuanto es la que forma a los ciudadanos que la sociedad requiere (García, 2016), (Onopriienko, Onopriienko, Petrushenko y Onopriienko, 2021). En el Perú, el Ministerio de educación ha lanzado el Proyecto Educativo Nacional al 2036, el cual se enfoca en el logro de una ciudadanía plena que implica entre otras cualidades y valores: el respeto a la naturaleza y la búsqueda del bien común (Begum et al, 2021). Además, está el enfoque transversal ambiental en el Programa Curricular Nacional de la Educación Básica - CNEB, para promover la ecoeficiencia en los estudiantes, de forma que sean los autores de la identificación y resolución de la problemática ambiental que se les presente (Yan-Hong y Yi-Huang, 2020). Problemática muy variada como variada es la cultura en los diferentes escenarios geográficos de nuestro País (Ministerio de Educación - MINEDU, 2021), (Pellín, Cuevas, Rodríguez y Gabarda, 2021).

Estos aprendizajes, necesarios para la construcción de la Ciudadanía Ambiental requiere adecuadas metodologías educativas (Monte y Reis, 2021). La realidad es que los docentes 
no logran situar estas propuestas en la planificación curricular, les hace falta las herramientas pedagógicas para asumir el enfoque ambiental en su práctica diaria (TovarGálvez, 2017). En este trabajo se propuso la estrategia formativa los Ecotalleres e investigar ¿De qué manera la aplicación de Ecotalleres influyen en la mejora del conocimiento de la planificación curricular en las docentes de inicial?

El trabajo de investigación es relevante dado que generó datos reales sobre las necesidades de las maestras, de herramientas en la planificación curricular para fomentar en los estudiantes la sensibilidad ante la problemática ambiental, las cuales irá mejorando con la formación en servicio, a través de diversas estrategias didácticas de esta naciente "Ecopedagogía", como los Ecotalleres y modelando con sus propias actitudes (Georgiou, Hadjichambis, y Hadjichambi, 2021).

El objetivo general fue Implementar, aplicar y evaluar los Ecotalleres para la mejora del conocimiento sobre planificación curricular con enfoque ambiental en docentes de inicial y, como objetivos específicos: Implementar Ecotalleres sobre planificación curricular con enfoque ambiental para docentes del nivel inicial; Aplicar Ecotalleres sobre planificación curricular con enfoque ambiental en las docentes de inicial; Describir el nivel de conocimiento sobre planificación curricular con enfoque ambiental antes de la aplicación de los Ecotalleres; Describir el nivel de conocimiento de la planificación curricular con enfoque ambiental después de la aplicación de los Ecotalleres y; Comparar el nivel de conocimiento sobre planificación curricular con enfoque ambiental de las docentes de inicial, antes y después de la aplicación de los Ecotalleres.

Los objetivos se propusieron comprobar las hipótesis: HI: La aplicación de los Ecotalleres influyen significativamente en el nivel de conocimiento de la planificación curricular con enfoque ambiental de las educadoras del nivel educativo inicial en la provincia del Santa - 2021. Y HO: La aplicación de los Ecotalleres no influyen en el nivel de conocimiento de la planificación curricular con enfoque ambiental de las educadoras de inicial.

El tema de investigación ha sido abordado desde diversas perspectivas y en diferentes ámbitos de acción, así tenemos en el ámbito internacional, la tesis doctoral de Lemos (2018) Una propuesta Pedagógica de Educación Ambiental, desde la perspectiva Bio céntrica basada en la cosmovisión de los nativos Cubeos, Jiw, Piratapuyos y Tuyucas en Bogotá D.C. en el que Lemos concluyó que los grandes saberes ancestrales de las comunidades del estudio no son conocidas y por lo tanto no son valoradas. El autor 
recomendó que la educación ambiental que se promueva, responda a la cosmovisión de las comunidades para generar conciencia ambiental eficazmente.

Lopera (2019) en su Tesis Doctoral Educación ambiental y profesionalización docente: diseño y reiteración de un modelo formativo; concluye que la formación de docentes en Educación Ambiental implica promover acciones delimitadas y planeadas para aulas reales en el contexto particular de la institución educativa y su aplicación aduce procesos sistemáticos de investigación para fortalecer no solamente los conocimientos curriculares y didácticos sino también la investigación.

Agreda (2016) en su tesis doctoral Capacitación docente para una formación humanizadora; llegó a las conclusiones que, las entidades formadoras deben determinar los valores que transmitirán en su currículo y en toda circunstancia, la formación docente se encuentra transitando de un paradigma que deshumaniza la labor a una nueva visión del docente como formador de conciencias en base a los pilares educativos y las demandas sociales $\mathrm{y}$, debe ser comprensiva de la integralidad del ser humano, los valores que requiere, la motivación y significatividad.

Kleinert (2020) en su tesis doctoral Capacitación continua de maestras de Preescolar y el soporte de directivos en la transferencia del aprendizaje. Universitat Autónoma de Barcelona, concluyó que, el apoyo de las directoras para favorecer la transferencia de aprendizajes de las docentes que se capacitan continuamente, es débil y recomienda que se continúe capacitando en su función a las involucradas en la investigación.

Albarracín (2017) en su Tesis Doctoral: Cultura ambiental en la administración escolar: Un estudio en el nivel preescolar de las escuelas públicas en Nariño, Bogotá - Colombia. Alcanzó las siguientes conclusiones: desde la gestión de las escuelas se logra promover la formación de los maestros en el tema ambiental y en los niños la formación integral, también halló una fuerte relación entre la educación ambiental del preescolar y la gestión institucional; por lo que es importante fortalecer la planificación para promover proyectos ambientales contextualizados, teniendo en cuenta los lineamientos de la formación preescolar y el involucramiento de las familias, estableciendo alianzas estratégicas con expertos. La autora también aporta una propuesta de gestión institucional-pedagógica para promover la educación ambiental en los preescolares.

En el ámbito nacional: La Tesis Doctoral de Rutti (2016) Educación ambiental de los maestros y la conciencia ambiental en el instituto Jaime Cerrón Palomino de Chongos 
Bajo, Chupaca - 2016; concluye que: se halló la directa relación y de carácter significativo entre la educación ambiental de los formadores del Instituto y la conciencia ambiental que se practica en el instituto.

La tesis de Vargas (2021) Los valores de los maestros como fundamento para promover la educación ambiental en la práctica pedagógica del nivel secundario; para obtener el grado de Doctor, produjo las siguientes conclusiones, los docentes ponen en práctica los valores personales en sus diferentes escenarios de vida, son referentes de valores y fomentan valores ambientales como la reflexión respecto a la problemática ambiental mediante la planificación de proyectos y sesiones, concursos ambientales; conocen parcialmente las políticas ambientales.

La tesis doctoral del Guevara (2020) Programa formativo docente para fortalecer la planificación curricular, concluye que, el programa aportó en el fortalecimiento de las capacidades de planificación de los profesores y recomienda a los profesionales de la UGEL que tomen en cuenta el programa en las acciones de capacitación; instando a los docentes a esforzarse en mejorar sus capacidades con esta herramienta.

La tesis doctoral de Quiroz (2015) Reforzamiento de las capacidades docentes realizado por la Municipalidad de Pisco - Ica, en la que el autor concluye que, el programa de capacitación dado por el municipio fortaleció las prácticas docentes en lo relacionado a la planificación y, en cuanto al tratamiento en las dimensiones: necesidades pedagógicas, necesidades humanas y necesidades investigativas tuvo una amplia aceptación entre la comunidad docente. Por lo que recomienda continuar con estrategias similares teniendo en cuenta la pertinencia, la reflexión continua, fomentar la investigación y establecer alianzas para seguir ejecutándolos.

En el contexto de la Región Ancash, Ita (2016) en su tesis doctoral: La Gestión en los proyectos ecológicos y sostenibles para el desarrollo de la cultura ecológica en escolares del sexto de primaria de la I.E. "Fe y Alegría" N 19 - Huaraz; arribó a las conclusiones que, existe un alto nivel de relación entre gestionar proyectos ecológicos y sostenibles con el desarrollo de la cultura ecológica en escolares que, a mayor gestión de proyectos ecológicos sostenibles mayores serán los niveles de adquisición de actitudes y conductas ambientales en los alumnos. Y, recomendó ampliar la propuesta de proyectos ecológicos sostenibles fundamentados en la Ecopedagogía y desarrollados de forma transversal en la planificación curricular para alcanzar la ansiada ciudadanía ambiental de los escolares. 
En la Tesis Doctoral de Trinidad (2020) Formación de promotores de actitudes ambientales para las instituciones educativas Unidocentes Rurales de Huaraz; el autor concluye que la capacitación brindada a los promotores influye en la adquisición de actitudes ambientales en los escolares de la muestra, el grupo experimental superó ampliamente al grupo control en los aspectos cognitivo, conductual y afectivo. Por lo que el autor recomienda que se fortalezcan estas estrategias de capacitación a promotores ambientales y comunidad docente de Huaraz, así como realizar investigaciones similares en otros contextos rurales de la Región y el País con el soporte de la UNEGV.

Estos estudios en esencia buscaron construir una respuesta ante la crisis ambiental y se volvieron hacia la educación ambiental como la principal vía para acceder a la formación de las conciencias de los seres humanos. Una Educación Ambiental, que si bien es la respuesta a la problemática ambiental de los últimos decenios, pero se ha mantenido invariable (Caride y Meira, 2018). Sin embargo, requiere un cambio de paradigma, ya (Orgaz-Agüera, 2018).

Es entonces que se acude a la Ecopedagogía, movimiento que se impulsó en el marco de la primera asamblea mundial de la Carta de la Tierra con perspectivas educativas, convocado por la institución Paulo Freire, apoyado por el Consejo de la Tierra y la UNESCO, realizada en Brasil, entre el 23 y 26 de agosto de 1999 (López, 2017). La Ecopedagogía emergió como una alternativa efectiva para la formación del ciudadano ambiental planetario que se requiere (Marín, 2019). Sobre su etimología, Zimmerman (citado por López, 2017) explicaba que:

Ecopedagogía es una palabra compuesta de los términos griegos oikos, que significa: casa, habitad, y paidada-gogia, que significa: el arte de educar. Por lo que la Pedagogía es el arte de enseñar todo lo relativo al habitad de los seres vivos del planeta. (p.9)

\section{Sobre su conceptualización, Humberto (citado por López, 2017), enunciaba lo siguiente:}

Desde la perspectiva de la Ecopedagogía, se resalta la responsabilidad de la educación en la construcción de un modelo nuevo y diferente del mundo, donde se establezca la importancia capital de la resignificación del sentido de la formación educativa dirigida a la formación de un 
"ciudadano convivencial" que piense como habitante de una comunidad planetaria, que percibe el planeta como una totalidad única e identifique a los demás seres humanos que lo habitan como sus iguales; por lo tanto, desaparecerán las diferencias por razones geográficas, culturales, de raza, económicas y sociales. (p.10)

\section{Para Gutiérrez y Prado (2015) la educación que nos llevará al desarrollo es la Ecopedagogía y lo expresa así:}

“... una pedagogía para la promoción del aprendizaje del sentido de las cosas a partir de la vida cotidiana como una ruta que permita desarrollar nuevas relaciones con nosotros mismos, con la Tierra y con la humanidad. Se trata de una pedagogía que pone a la misma altura de todas las criaturas vivas, que reconoce a la Tierra como el planeta del que formamos parte; pues ella "no es un ser extraño y lejano, sino que es nuestra casa, el lugar en donde vivimos y convivimos" (p.100).

En ese sentido, la labor pedagógica debe alinearse con estos nuevos propósitos educativos para dar sostenibilidad a la planificación curricular y atender la problemática socioecológica priorizándola, teniendo en cuenta la intención pedagógica como los intereses de la comunidad educativa (Murga-Menoyo y Novo, 2017).

También suma lo expresado por Miranda y Bruno (2020) respecto a que toda educación ambiental debe promover a su vez la educación científica para que los estudiantes comprendan los contextos naturales y propongan actividades económicas con responsabilidad ciudadana donde comulguen las posiciones de las ciencias naturales y las ciencias sociales; en la esperanza de lograr desarrollar, efectivamente la conciencia ambiental del estudiante y, a la larga mejorar sus conductas ambientales y su contexto, actividades que se pueden desarrollar en los talleres (Charlie, Taat, Nordin y Saikim, 2021). En esa línea, se propusieron los Ecotalleres sobre Planificación Curricular con enfoque Ambiental, los que se desarrollaron en un periodo de ocho semanas y constituye la variable independiente de este trabajo de investigación. 


\section{Tabla 1}

Plan de Trabajo de los Ecotalleres

\begin{tabular}{|c|c|c|}
\hline $\mathbf{N}^{\circ}$ & Actividad & Metodología \\
\hline 1 & Reunión de Coordinación con los participantes & \multirow{11}{*}{$\begin{array}{c}\text { Aprendizaje } \\
\text { Situado } \\
\text { Participativo } \\
\text { Trabajo } \\
\text { colaborativo } \\
\text { Crítico reflexivo }\end{array}$} \\
\hline 2 & Aplicación del Pre Test & \\
\hline 3 & Ecotaller 1: Educación Ambiental & \\
\hline 4 & Ecotaller 2: Educación Ambiental: Proyecto de Enfoque Ambiental Integrado (PEAI) & \\
\hline 5 & Ecotaller 3: Cambio climático: Glocalidad & \\
\hline 6 & Ecotaller 4: Cambio climático: Cuidado del aire y del suelo & \\
\hline 7 & Ecotaller 5: Ecoeficiencia: Gestión de residuos sólidos y las 3R & \\
\hline 8 & Ecotaller 6: Ecoeficiencia: Emprendimiento socio ambiental & \\
\hline 9 & Ecotaller 7: Salud & \\
\hline 10 & Ecotaller 8: Riesgos y desastres & \\
\hline 11 & Aplicación del post Test. & \\
\hline
\end{tabular}

Y se fundamentaron en las características didácticas de los talleres, la oportunidad que brindan de aprender haciendo, proponiendo (Paula-Acosta, Pérez-López y SierraSocorro, 2019). El fomento del Aprendizaje Situado, la reflexión, dando espacios para la interacción y expresión de los sentimientos, experiencias e ideas, en busca de una solución común estableciendo compromisos, propuestas, etc. (Fernández-Hernández, Manchón-Reina y Castillo-Escalona, 2018).

Vygotsky, citado por Gómez y Acosta (2017) sustenta la importancia de la comunicación social en la interacción y reflexión, donde es el lenguaje el que permite constantemente la reorganización mental y la construcción del significado. También agrega que se necesita que los aprendices estén motivados para aprender en interacción. Lo relacionamos con lo que afirman Gómez y Acosta (2017) que los talleres: son espacios de aprendizaje grupal en los que se aterriza la teoría partiendo de la información que traen los participantes, reiterando el valor de la interacción siempre que se favorezca un clima de respeto y apertura. Considerando sus necesidades de fortalecimiento, inquietudes y expectativas agrega Preitz (2021). Así pues, contextualizando, los Ecotalleres son altamente participativos, dinámicos, variados e integradores (Maya, 2016).

Y, al ser las maestras del nivel inicial, las participantes de los Ecotalleres, las estrategias didácticas se basaron en la andragogía. En palabras de Knowles, el padre de la Andragogía, citado por Castillo (2018) “...la andragogía es un conjunto de principios fundamentales sobre el aprendizaje de adultos que se aplica a todas las situaciones de tal 
aprendizaje... la andragogía está orientada hacia la educación para adultos..." (p. 65). Así también, el Dr. Adam, citado por Castillo (2018) explica que, en base a las particularidades de los adultos, la diferencia entre la pedagogía y la andragogía consiste en que mientras la primera es un proceso de aprendizaje dirigida por el pedagogo, en la segunda es un proceso de orientación del aprendizaje autónomo del participante, a cargo de un facilitador, regido por dos principios elementales: la horizontalidad y la participación. Con respecto a la evaluación, esta responsabilidad recae en el participante por lo que es necesario que se den espacios para la autoevaluación, la coevaluación y heteroevaluación del andragogo (Castillo, 2018).

Con respecto a la variable dependiente, Planificación Curricular, principal tarea de la labor docente, como se estipula en el Marco de Buen Desempeño Docente - MBDDoc. (MINEDU 2012). Se realiza anualmente, previo al inicio de sus labores con los estudiantes, llamada también planificación a largo plazo y, en periodos más cortos: mensuales, quincenales: planificación a corto plazo. Toda planificación se soporta en el diagnóstico, así como las competencias y enfoques transversales establecidos en el Programa Curricular para el nivel y ciclo correspondiente. Es flexible, abierta y dinámica, por lo que se dice que constituye una hipótesis que se va precisando durante su ejecución. (MINEDU, 2016a). La planificación a corto plazo, se deriva de la Planificación Anual, se ejecuta a través de la Experiencia de Aprendizaje, requiere la secuencia organizada de actividades que desafiarán a los niños para la resolución de una situación problemática o el logro de un objetivo, prevé los recursos, medios y materiales, los cuales deben tomarse del contexto inmediato de los niños (MINEDU, 2016a). Se diseña a la luz de los Principios del nivel Inicial: Respeto, Seguridad, Salud, Autonomía, Movimiento, Juego libre y Comunicación (MINEDU, 2016b).

A la par de la Planificación Curricular, también se diseña la evaluación con enfoque formativo, es la evaluación "para seguir aprendiendo", en ella se valoran los logros alcanzados por los estudiantes para plantearles nuevas estrategias que les ayude a seguir avanzando de manera pertinente (Anijovich, 2017). La evaluación con enfoque formativo, requiere que los docentes asuman nuevos roles de acompañamiento y de apoyo permanente a los procesos de aprendizaje de los estudiantes (Ravela, Picaroni y Loureiro, 2017). El documento normativo de estos procesos de Planificación Curricular es el CNEB que sustenta el enfoque de competencias. En ese punto, parafraseando a Masciotra (2017) 
se refiere al actuar de un sujeto en una situación real, la cual opera y desarrolla con las herramientas que posee, los conocimientos, capacidades y habilidades que acumuló en otro escenario y aplica en una nueva situación, adecuándolas. Este enfoque observa al estudiante como un ser integral multidimensional: físico, biológico, psíquico, cultural, histórico y social a la vez; tomando conocimiento, como conciencia, de la complejidad de su identidad individual (sus particularidades) y de su identidad como ser humano. El CNEB, también orienta la implementación de las condiciones: espacios, materiales y el rol mediador del adulto (MINEDU, 2016a).

\section{ESTRATEGIAS METODOLÓGICAS O MATERIALES Y MÉTODOS}

La investigación realizada fue de tipo Aplicada, Experimental. Privitera, Fleiss y O’brien (citados por Hernández-Sampieri y Mendoza, 2018) manifiestan que se refiere a aquellos estudios en los cuales se realiza una intervención: la variable independiente, una manipulación intencional de la que posteriormente se analizan los efectos y consecuencias que éstas causan sobre las variables dependientes en una determinada situación de investigación. De diseño Pre experimental, se trabajó con un único grupo, en estos estudios se tiene un mínimo nivel de control e incluye una pre prueba, así como, una post prueba (Hernández-Sampieri y Mendoza, 2018). Este diseño se grafica de la siguiente manera:

\section{GE: O1--- X--- 02 --- P}

\section{Donde:}

GE: Grupo experimental

O1: es el pre test aplicado (prueba de entrada)

$\mathbf{X}$ : es la variable experimental (Aplicación de los Ecotalleres)

O2: es el post test aplicado (prueba de salida).

P: Es la Propuesta

La población de estudio la constituyeron los 4413 docentes de Educación Básica Regular (EBR) de las Instituciones Educativas Públicas de la Provincia del Santa. La muestra estuvo integrada por las docentes de educación Inicial del II Ciclo de EBR que atienden a los estudiantes de 3, 4 y 5 años de edad. El muestreo de determinó de forma no probabilístico, por conveniencia, a criterio del investigador, debido a que se necesitaba la disposición de las docentes para participar en los Ecotalleres; contando con la 
confirmación de 65 docentes de 13 Instituciones Educativas de 08 distritos de la Provincia del Santa.

\subsection{Técnicas e instrumentos}

Tabla 2

Técnica e instrumento

\begin{tabular}{|l|l|}
\hline \multicolumn{1}{|c|}{ Técnica } & \multicolumn{1}{c|}{ Instrumento } \\
\hline $\begin{array}{l}\text { Encuesta a } \\
\text { docentes }\end{array}$ & $\begin{array}{l}\text { Cuestionario sobre la Planificación Curricular con Enfoque } \\
\text { Ambiental, de 26 ítems, tipo de respuesta: opción múltiple. }\end{array}$ \\
\hline
\end{tabular}

El cuestionario constó de 26 preguntas agrupadas en cuatro dimensiones: Enfoque ambiental transversal al CNEB, los principios del nivel inicial, planificación a largo plazo y experiencias de aprendizaje (planificación a corto plazo) y se aplicó a través de un formulario virtual.

Validez del instrumento: Fue realizado por dos expertos profesionales, educadores del Nivel Inicial de la EBR (Carrasco, 2019).

Confiabilidad de los instrumentos: Se aplicó el instrumento a 15 docentes del nivel inicial, no pertenecientes a la muestra de estudio y los resultados se verificaron con el Alfa de Conbrach, obteniéndose un nivel de confiabilidad de: 0.92 (Abanto, 2016).

\subsection{Procedimientos}

Se envió un oficio de invitación a los directivos de las Instituciones Educativas que han manifestado su deseo de participar en la aplicación de la propuesta de investigación, dándoles la información sobre los detalles de su participación, en los Ecotalleres, se recibió los documentos de confirmación de su participación. Se les aplicó a las participantes un pre test, de forma anónima, para determinar el nivel de conocimiento de la planificación con enfoque ambiental que realizan, se ejecutaron los Ecotalleres una vez a la semana durante ocho semanas, en horario consensuado con las participantes al cabo del cual se les aplicó el post test para comparar con el anterior resultado y verificar la efectividad de la propuesta.

\section{Método de análisis de datos}

Se recurrió a la estadística descriptiva, luego de la aplicación del programa MS Excel 2019, con el que se ordenaron los datos que se obtuvieron de la aplicación del instrumento, se tuvo una base de datos, las que se presentaron en tablas, con los 
resultantes: media, desviación estándar, en el que se pudo ver el comportamiento de los datos; se compararon las medias obtenidas en el pre y post test, para comprobar si se observaba la mejora (Hernández-Sampieri y Mendoza, 2018).

\section{RESULTADOS Y DISCUSIÓN}

\section{Tabla 3}

Prueba de hipótesis del pre test y post test del nivel de conocimiento de planificación curricular con enfoque ambiental.

\begin{tabular}{|c|c|c|c|c|c|}
\hline & $\begin{array}{c}\text { Postest- } \\
\text { Pretest } \\
\text { Educación } \\
\text { ambiental }\end{array}$ & $\begin{array}{c}\text { Postest- } \\
\text { Pretest } \\
\text { Principios } \\
\text { del nivel } \\
\text { Inicial }\end{array}$ & $\begin{array}{c}\text { Postest-Pretest } \\
\text { Planificación } \\
\text { Curricular } \\
\text { Anual }\end{array}$ & $\begin{array}{c}\text { Postest- } \\
\text { Pretest } \\
\text { Experiencia } \\
\text { de } \\
\text { Aprendizaje } \\
\text { EdA }\end{array}$ & V $_{\text {ca-post }}$ \\
\hline$Z$ & $-6,537^{\mathrm{b}}$ & $-4,595^{\mathrm{b}}$ & $-6,719^{\mathrm{b}}$ & $-5,622^{\mathrm{b}}$ & $-6,942^{\mathrm{b}}$ \\
\hline $\mathrm{P}$ &, 000 &, 000 &, 000 &, 000 &, 000 \\
\hline
\end{tabular}

Fuente: Data del pre - post test de Conocimiento sobre Planificación Curricular (2021)

${ }^{a}$ Prueba de rangos con signo de Wilcoxon.

${ }^{\mathrm{b}} \mathrm{Se}$ basa en rangos negativos.

\section{Interpretación:}

Los resultados presentados en la Tabla 4, demostraron una diferencia significativa de los datos obtenidos, tanto en las dimensiones como en la variable entre el post test y el pre test empleado para la presente investigación, evidenciando los valores de $\mathrm{P}=0.000$ los cuales son menores 0.05 . por lo que se rechaza la hipótesis nula y se acepta la hipótesis del investigador: la aplicación, de los Ecotalleres influyen significativamente en el nivel de conocimiento de la planificación curricular con enfoque ambiental de las educadoras del nivel educativo inicial en la provincia del Santa - 2021.

\section{CONCLUSIÓN O CONSIDERACIONES FINALES}

\section{Luego del trabajo realizado, con respecto a los objetivos trazados:}

Primero: Se concluyó de manera exitosa con la implementación de los Ecotalleres sobre planificación curricular con enfoque ambiental para las maestras del nivel inicial considerando la construcción colaborativa de propuestas de planificación en el marco del CNEB, el Programa Curricular y, a la luz de los principios del nivel inicial, con espacios para la autoevaluación, la coevaluación y evaluación de la facilitadora. Las estrategias se alinearon a las necesidades detectadas en el pre test y las condiciones de su ejecución se 
consensuó con las participantes quienes, al finalizar, manifestaron un compromiso de 98\% que denota satisfacción con el formato de los Ecotalleres.

Segundo: Se concluyó que, las estrategias aplicadas los Ecotalleres, lograron sensibilizar significativamente a las maestras, quienes comprendieron la transversalidad del Enfoque ambiental en el CNEB, sus características, valores, y lo evidencian con 95\% de asistencia continua, así como un $62 \%$ de participantes en el nivel Logrado en los trabajos colaborativos.

Tercero: Se describió exitosamente el nivel de conocimiento de la planificación curricular con enfoque ambiental en las docentes de educación inicial de la Provincia del Santa, antes de la aplicación de los Ecotalleres, el cual mostraba las necesidades de las docentes y no se había detenido a analizar oportunamente constituyéndose en una labor pendiente e importante de atender como lo muestra el grupo mayoritario de $83 \%$ de docentes en el nivel En inicio.

Cuarto: Se describió exitosamente el nivel de conocimiento de la planificación curricular con enfoque ambiental después de la aplicación de los Ecotalleres, en las docentes de educación inicial, pudiéndose determinar los niveles al detalle en los diferentes niveles: en Destacado: 27.69\%, en Esperado: 33.85\%, En Proceso: $32.31 \%$ y en Inicio: $6.15 \%$, además de los resultados obtenidos por las maestras en los trabajos colaborativos y encuestas cuyo resumen muestra un $62 \%$ en nivel Logrado.

Quinto: Se logró comparar exitosamente el nivel de conocimiento sobre planificación curricular con enfoque ambiental de las docentes de inicial de la provincia del Santa, antes y después de la aplicación de los Ecotalleres, hallándose notables diferencias: como el del grupo mayoritario de $83 \%$ que antes de la aplicación se encontraban en el nivel de inicio avanzaron a los niveles superiores de proceso, esperado y destacado ya que después de los Ecotalleres en este nivel solo quedaron $6.15 \%$; el $15 \%$ que antes se encontraba En proceso, después avanzó a 32.31\%; el 2\% que antes se encontraban en el nivel Esperado, después alcanzó el 33.85\% y en el nivel destacado antes de los Ecotalleres no teníamos ninguna docente, después de ellos, alcanzamos el $27.69 \%$.

Sexto: Y dando respuesta a la pregunta de investigación se concluyó que, se rechazó la hipótesis nula y se aceptó la hipótesis de investigación que los Ecotalleres influyen significativamente en el nivel de conocimiento de la planificación curricular con enfoque ambiental en las docentes del nivel inicial, evidenciando su efectividad; dado que, el 
análisis de la Prueba de rangos con signos de Wilcoxon en la tabla 11, evidencia los valores de $\mathrm{P}=0.000$, menores a 0.05 .

\section{LISTA DE REFERENCIAS}

Agreda, A. (2016) Capacitación docente para una formación humanizadora (Tesis doctoral). Universidad de Carabobo, Bárbula.

Albarracín, S. (2017) Cultura ambiental en la gestión institucional: Un estudio de caso en el nivel preescolar de colegios oficiales en Antonio Nariño (Tesis doctoral). Universidad Santo Tomás, Bogotá, D.C.

Anijovich, R. (2017). La evaluación como oportunidad. Buenos Aires, Argentina: Paidós.

Arteaga-Cruz, E., Mukhopadhyay, B., Shannon, S., Nidhi, A. y Jailer, T. (2021) Connecting the right to health and anti-extractivism globally. Saúde Debate, 44(1), 100-108. doi: 10.1590/0103-11042020S108.

Begum, A., Jingwei, L., Haider, M., Ajmal, M., Khan, S. y Han, H. (2021). Impact of environmental moral education on pro-environmental behaviour: Do psychological empowerment and islamic religiosity matter? International Journal of Environmental Research and Public Health, 18(4), 1-19. doi:10.3390/ijerph18041604

Caride, J. y Meira, P. (2018). Del ecologismo como movimiento social a la educación ambiental como construcción histórica. Historia de la educación, 37(1), 165-197. doi: 10.14201/hedu201837165197.

Carrasco, S. (2019) Scientific Investigation Methodology. Methodological guidelines to design and develop the research project. Lima, Perú: San Marcos E.I.R.LTDA.

Castillo, F. (2018). Andragogía, andragogos y sus aportaciones. Voces De La Educación, 3(6), 64-76.

Charlie, E., Taat, M., Nordin, M. y Saikim, F. (2021) The Impact of Environmental Education (EE) on the Society's Awareness, Responsibility, and Attitude towards the Development of a Lifelong Attitude of Pro-Conservation Behaviour in Kota Kinabalu, Sabah. Earth and Environmental Science, 736 (2021), 012012. doi:10.1088/1755-1315/736/1/012012

Fernández-Hernández, J., Manchón-Reina, D. y Castillo-Escalona, J. (2018) Environmental culture in the training of the education professionals: an 
experience from the subject physical education. Olimpia. Revista de la Facultad de Cultura Física de la Universidad de Granma, 15(48).

García, M. (2016) Deforestation: a practice that kills our biodiversity. Production clean, 11(1), 161-168. doi: $10.22507 / \mathrm{pml} . v 11 \mathrm{n} 2 \mathrm{a} 13$

Georgiou, Y., Hadjichambis, A. y Hadjichambi, D. (2021) Teachers' Perceptions on Environmental Citizenship: A Systematic Review of the Literature. Sustainability, 13, 2622. doi: 10.3390/su13052622

Gómez, A. y Acosta, R. (2017). El taller pedagógico interactivo-reflexivo para la formación y superación profesional de profesores de lenguas. MENDIVE. Recuperado de: http://mendive.upr.edu.cu/index.php/MendiveUPR/article/view/1013

Guevara, Y. (2020) Programa formativo docente para fortalecer la planificación curricular. (Tesis doctoral) Universidad Cesar Vallejo, Chiclayo, Perú.

Gutiérrez, F. y Prado, C. (2015). Ecopedagogía y ciudadanía planetaria. Ciudad de México, México: De La Salle Editores.

Hernández-Sampieri, R. y Mendoza, C. (2018) Metodología de la Investigación: Las rutas cuantitativa, cualitativa y mixta. Ciudad de México, México: Mc Graw-Hill Interamericana.

Huynh, H. (2020) Environmental education for the sustainable development of suburban communities in Ho Chi Minh City. University of Vietnam, 234(1). doi:10.1051/e3sconf/202123400057

Ita, O. (2016). Gestión de proyectos ecológicos sostenibles y su relación con el desarrollo de cultura ambiental en estudiantes del sexto grado de educación primaria de la I.E. "Fe y Alegría” $N^{\circ} 19$ - Huaraz (Tesis doctoral). Universidad Cesar Vallejo, Chimbote, Perú.

Kleinert, E. (2020) Capacitación continua de maestras de Preescolar y el soporte de directivos en la transferencia del aprendizaje (Tesis doctoral). Universitat Autónoma de Barcelona, España.

Lemos J. (2018) Una propuesta Pedagógica de Educación Ambiental, desde la perspectiva Biocéntrica (Tesis doctoral) Universidad Santo Tomás, Bogotá, Colombia. 
Lopera, M. (2019) Educación ambiental y profesionalización docente: diseño y reiteración de un modelo formativo (Tesis Doctoral). Universidad de Valladolid, España.

López, L. (2017) Ecopedagogía. Bogotá, Colombia: Areandino.

Marín, T. (2019) Ecopedagogy in the construction of citizenship a review. Social Innova Sciences, 1(1).

Masciotra, D. (2017) La compétition: Entre savoir agir et agir réel. Perspective de l'énaction. Éthique publique, 19(1), 1-15.

Maya, A. (2016) El taller Educativo. ¿Qué es? Fundamentos, cómo organizarlo y dirigirlo, cómo evaluarlo. Magisterio Editorial, 113-136.

MINEDU (2012) Marco del Buen Desempeño Docente. Lima, Perú: Ministerio de educación.

MINEDU (2016a) Currículo Nacional de la Educación Básica. Lima, Perú: Ministerio de educación.

MINEDU (2016b) Entorno educativo de calidad en Educación Inicial - Guía para docentes del Ciclo II "Conocer para planificar y mejorar. Lima, Perú: Ministerio de educación.

MINEDU (2021) Proyecto Educativo Nacional al 2036. Lima, Perú: Ministerio de educación.

Miranda, D., y Bruno, I. (2020). A abordagem socioambiental na educação em ciências como caminho para a construção da cidadania na sociedade de Risco. Pesquisa em Educação em Ciências. Revista Ensaio - Belo Horizonte. 22(1). doi.org/10.1590/1983-21172020210138.

Monte, T. y Reis, P. (2021) Design of a Pedagogical Model of Education for Environmental Citizenship in Primary Education. Sustainability, 13, 6000. doi.org/10.3390/su13116000

Murga-Menoyo, M. (2018). La Formación de la Ciudadanía en el Marco de la Agenda 2030 y la Justicia Ambiental. Revista Internacional de Educación para la Justicia Social, 7(1), 37-52. doi: 10.15366/riejs2018.7.1.002.

Onopriienko, K., Onopriienko, V., Petrushenko, Y. y Onopriienko, I. (2021). Environmental education for youth and adults: A bibliometric analysis of research. 234(2) doi:10.1051/e3sconf/202123400002 
Orgaz-Agüera, F. (2018). Educación ambiental: concepto, origen e importancia. El caso de república dominicana. Revista DELOS Desarrollo Local Sostenible, 31. Recuperado de https://www.eumed.net/rev/delos/31/franciscoorgaz.html//hdl.handle.net/20.500.11763/delos31francisco-orgaz

Paula-Acosta, C., Pérez-López, J. y Sierra-Socorro, J. (2019) Environmental education with an integrative approach. An experience in the initial training of mathematics and physics teachers. Educare, 23(1), 181-202. doi: 10.15359/ree.23-1.10

Pellín, A., Cuevas, N., Rodríguez, A. y Gabarda, V. (2021) Promotion of Environmental Education in the Spanish Compulsory Education Curriculum. A Normative Analysis and Review. Sustainability, 13,24-69, doi.org/10.3390/su13052469

PNUMA (2019). Informe sobre la disparidad en las emisiones de 2019. ONU Programa de las Naciones Unidas para el Medio Ambiente, Nairobi. Recuperado de https://wedocs.unep.org/bitstream/handle/20.500.11822/30798/EGR19ESSP.pdf ?sequence $=17$

Preitz, T. (2021): Consistency in study programme planning and the complexity of curriculum logics. Teaching in Higher Education, 237960 doi: $10.1080 / 13562517.2021 .1931838$

Quiroz, L. (2015) Reforzamiento de las capacidades docentes realizado por la Municipalidad de Pisco - Ica (Tesis doctoral). Universidad Peruana Cayetano Heredia, Lima, Perú.

Ravela, P., Picaroni, B. y Loureiro, G. (2017) ¿Cómo mejorar la evaluación en el aula? Reflexiones y propuestas de trabajo para docentes. Ciudad de México, México: Grupo Mayo Editores.

Rutti, J. (2016) Educación ambiental de los maestros y la conciencia ambiental en el instituto Jaime Cerrón Palomino de Chongos Bajo, Chupaca (Tesis doctoral) Universidad Cesar Vallejo, Perú.

Sarria, R. \& Gallo, J. (2016) The big environmental problem of the plastic waste: Microplastics. Journal de Ciencia e Ingeniería. 8(1). Recuperado de http://jci.uniautonoma.edu.co/2016/2016-3.pdf

Silva, M. y Passos, M. (2018). Discurso de autores da paisagem do Pantanal da Nhecolândia. Mercator, 17(17015). doi:10.4215/rm2018.e17015 
Tovar-Gálvez, J. (2017). Pedagogia ambiental e didática ambiental: tendências na educação superior. Revista Brasileira de Educação, 22(69), 519-538. Recuperado de: https://www.redalyc.org/articulo.oa?id=27553036011

Trinidad, N. (2020) Formación de promotores de actitudes ambientales para las instituciones educativas Unidocentes Rurales de La Libertad-Huaraz (Tesis doctoral). Universidad Enrique Guzmán y Valle. Lima, Perú.

Vargas, M. (2021) Los valores de los maestros como fundamento para promover la educación ambiental en la práctica pedagógica del nivel secundario (Tesis doctoral). Universidad Cesar Vallejo, Lima, Perú.

Yan-Hong, Y. y Yi-Huang Sh. (2020) Environmental Education for Children in Taiwan: Importance, Purpose and Teaching Methods. Universal Journal of Educational Research, 8(4): 1572-1578. doi: 10.13189/ujer.2020.080451 